\title{
ORIGINAL ARTICLE AAV-mediated photoreceptor transduction of the pig cone-enriched retina
}

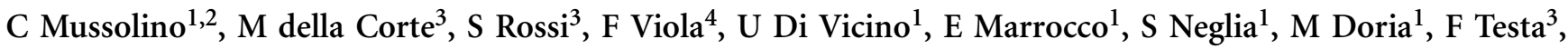

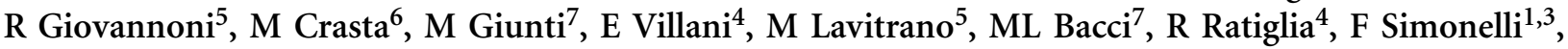 \\ A Auricchio $^{1,8}$ and EM Surace ${ }^{1}$
}

Recent success in clinical trials supports the use of adeno-associated viral (AAV) vectors for gene therapy of retinal diseases caused by defects in the retinal pigment epithelium (RPE). In contrast, evidence of the efficacy of AAV-mediated gene transfer to retinal photoreceptors, the major site of inherited retinal diseases, is less robust. In addition, although AAV-mediated RPE transduction appears efficient, independently of the serotype used and species treated, AAV-mediated photoreceptor gene transfer has not been systematically investigated thus so far in large animal models, which also may allow identifying relevant species-specific differences in AAV-mediated retinal transduction. In the present study, we used the porcine retina, which has a high cone/rod ratio. This feature allows to properly evaluate both cone and rod photoreceptors transduction and compare the transduction characteristics of AAV2/5 and 2/8, the two most efficient AAV vector serotypes for photoreceptor targeting. Here we show that AAV2/5 and 2/8 transduces both RPE and photoreceptors. AAV2/8 infects and transduces photoreceptor more efficiently than AAV2/5, similarly to what we have observed in the murine retina. The use of the photoreceptor-specific rhodopsin promoter restricts transgene expression to porcine rods and cones, and results in photoreceptor transduction levels similar to those obtained with the ubiquitous promoters tested. Finally, immunological, toxicological and biodistribution studies support the safety of AAV subretinal administration to the large porcine retina. The data presented here on AAV-mediated transduction of the cone-enriched porcine retina may affect the development of gene-based therapies for rare and common severe photoreceptor diseases.

Gene Therapy (2011) 18, 637-645; doi:10.1038/gt.2011.3; published online 17 March 2011

Keywords: AAV; retina; large animal model; photoreceptor transduction; biodistribution; pig

\section{INTRODUCTION}

The recent promising results of gene therapy in patients with leber congenital amaurosis $\left(L C A^{1}\right)$, the most severe form of inherited childhood blindness, supports the safety and efficacy of retinal gene transfer through adeno-associated viral $(\mathrm{AAV})$ vectors. $^{2-7}$ The therapeutic success behind the LCA trials is mainly because of two factors: (1) the availability of vectors that enable an efficient transduction of the retinal pigment epithelium (RPE), the target cells in LCA2, the specific $L C A$ form selected for the trials; and (2) the availability of a relevant $L C A 2$ large animal model. The consistency of the data relative to the safety and efficacy of gene transfer obtained in LCA2 models as diverse as mice, ${ }^{8} \operatorname{dogs}^{9}$ and humans, strongly supports the concept that AAV-mediated RPE-specific gene transfer may be extended from animal models to humans.

Owing to its simple monolayer organization, and its functional and biological properties, ${ }^{10}$ the RPE is efficiently transduced by virtually any AAV vector serotypes tested. ${ }^{11,12}$ However, the majority of inherited forms of retinal diseases affect rod and/or cone photoreceptors, whose transduction poses greater difficulties compared with the RPE. To date, only a few AAV serotypes have been shown to efficiently target animal model photoreceptors and a systematic side-by-side comparison of large animal photoreceptor transduction by different AAV serotypes is still lacking. ${ }^{12}$ Notwithstanding, the data available thus far suggest that AAV2/5 and AAV2/8 efficiently transduce photoreceptors in rodents, ${ }^{11,13-16}$ whereas AAV2/2, AAV2/5 and AAV2/8, independently tested, appear to be efficient in transducing photoreceptors in dogs, ${ }^{9,17,18}$ and non-human primates (NHP). ${ }^{19-22}$ In addition, owing to limitations of the experimental models used, the difference between rod and cone transduction remains difficult to assess. In particular, the distribution and number of cone photoreceptors in the murine retina represents a major limitation to precisely define their transduction, whereas the primate retina, which generally is considered dominated by cones, has on average a relatively low cone/rod ratio $\left(1: 20\right.$ in humans $\left.{ }^{23}\right)$. The porcine eye presents a high cone/rod ratio distributed throughout the retina (an overall average cone/rod ratio of 1:7) and a large cone-rich streak region encompassing the horizontal meridian where the cone/rod ratio reaches 1:3/1:5. ${ }^{24}$ Thus, pigs represent an ideal large animal model to study cone transduction characteristics also compared with the NHP retina, in which the cone/rod ratio is low (1/15) beyond the small foveal area. ${ }^{23}$ The abundance of both rods and cones makes the porcine retina particularly amenable to determine the transduction of the single

${ }^{1}$ Telethon Institute of Genetics and Medicine, Naples, Italy; ${ }^{2}$ SEMM-European School of Molecular Medicine, Naples site, Italy; ${ }^{3}$ Department of Ophthalmology, Second University of Naples, Naples, Italy; ${ }^{4}$ U Oculistica, Fondazione IRCCS, Ospedale Maggiore Policlinico, Mangiagalli e Regina Elena, University of Milan, Milan, Italy; ${ }^{5}$ Department of Surgical Sciences. University of Milano-Bicocca, Monza, Italy; ${ }^{6}$ Visionvet, Eye Clinic for Animal, Bologna, Italy; ${ }^{7}$ Department of Veterinary Morphophysiology and Animal Production, University of Bologna, Bologna, Italy and ${ }^{8}$ Medical Genetics, Department of Pediatrics, 'Federico II' University, Naples, Italy

Correspondence: Dr EM Surace or A Auricchio, Telethon Institute of Genetics and Medicine. Via P Castellino, 111.80131 Napoli, Italy.

E-mail: surace@tigem.it or auricchio@tigem.it

Received 6 January 2010; revised 16 August 2010; accepted 19 September 2010; published online 17 March 2011 
photoreceptor cells following subretinal administration of viral vectors. In addition, the porcine eye, more than that of other large mammals such as dogs and cows, ${ }^{25}$ shares many similarities with the human retina including, a nontapetal fundus with a holangiotic vascular pattern and the presence of retinal layers of similar thickness. The size and anatomy of the porcine eye globe, lens and cornea offer the possibility to test the feasibility of vitreo-retinal surgery techniques such as the subretinal injection of viral vectors. In addition, various porcine models of genetic and acquired retinal diseases are available. $^{26-28}$

We therefore sought to compare the transduction efficiency of $\mathrm{AAV} 2 / 5$ and $2 / 8$ in the porcine retina, as well as that of ubiquitous and photoreceptor-specific promoter elements.

\section{RESULTS AND DISCUSSION}

Subretinal administration of AAV vectors to the porcine retina

In this study, we injected 46 eyes subretinally either monolaterally or bilaterally from 11-week-old pure Large White female pigs ( $n=29$; live weight $=33 \pm 2 \mathrm{~kg}$ ). To target a region of the retina with a similar cone and rod photoreceptor content, AAV vectors were subretinally inoculated in the avascular nasal area of the posterior pole between the two main vascular arches (Figure 1a) in which cone density reaches 35000 cells per $\mathrm{mm}^{2}$ (see ref. 24). In the first set of experiments, in which our aim was to define the best serotype for photoreceptor transduction, we performed a traditional surgical procedure using 20gauge instrumentation for vitrectomy. In the subsequent experiments comparing promoter efficiency, we switched to a 23 -gauge technique with the aim of reducing surgical trauma providing numerous potential advantages, including faster wound healing, diminished conjunctival scaring with less post-operative redness and inflammation (see the Materials and Methods section). The AAV vector serotypes to be tested were either co-injected with AAV2/1-cytomegalovirus (CMV)-LacZ for normalization or injected alone (see below). We injected 1.0-2.0 $\times 10^{10}$ genome copies (GCs) of each vector in a total volume of $100 \mu \mathrm{l}$. To avoid lot-to-lot differences, two independent vector preparations per serotype/promoter construct were used. Subretinal vector administration resulted in the typical 'dome-shaped' retinal detachment (retinal bleb: Figure 1a). All animals underwent ophthalmologic examination within the first 3 days following surgery and at killing (6 weeks post-vector administration). Adverse events included inflammation, which spontaneously resolved: five irreversible retinal detachments $(10.8 \%$ of the treated eyes, all of which occurred at the beginning of experimentations when we were setting the surgical procedure), and 15 limited opacities of the posterior capsule in correspondence of the entry site of surgical instruments.

\section{AAV2/8 transduces porcine photoreceptors more efficiently than AAV $2 / 5$}

To evaluate the tropism of different AAV vector serotypes, following subretinal administration in pigs, we generated AAV2/5 and $2 / 8$ vectors expressing the enhanced green fluorescent protein (EGFP) from the CMV promoter (AAV2/5-CMV-EGFP; AAV2/8-CMVEGFP). Initially, nine Large White female pigs were subretinally co-injected with an AAV2/1-LacZ encoding for Escherichia coli $\beta$-Galactosidase $(\beta-\mathrm{Gal})$, to control over the potential variability of AAV vectors administration following subretinal surgery ${ }^{13}(n=5$ eyes
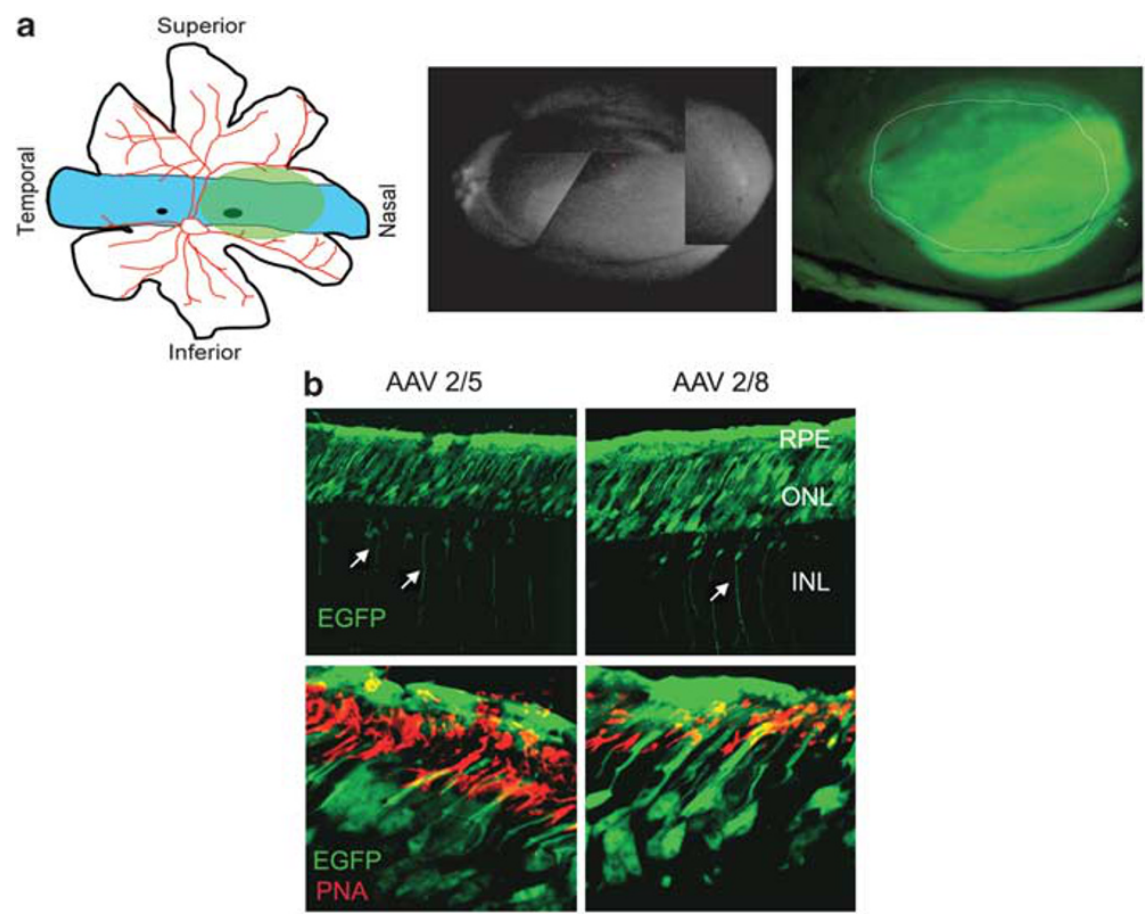

Figure 1 Tropism of AAV2/5 and AAV2/8 vectors subretinally injected in the porcine retina. (a, right panel) Schematic representation of the visual streak in the porcine retina with highest cone density (light blue; re-drawing from reference 24). The light green area represents the site and the extension of subretinal injection. (a, middle panel) Assembly of black and white fundus images showing dome-like retinal bleb following subretinal injection of $100 \mu$ l of AAV vector containing- solution. The asterisk indicates the injection site. (b) Confocal fluorescence microscopy analysis of retinal sections at 6 weeks after subretinal injection of AAV2/5-, or AAV2/8-CMV-EGFP. (b, upper panel) Outer retinal EGFP expression appearance ( $\times 20$ magnification). Arrows indicate EGFP-positive cells in the inner nuclear layer (INL). The images of the INL were taken at higher exposure than those of the outer retina. RPE, retinal pigment epithelium; ONL, outer nuclear layer (scale bar $=10 \mu \mathrm{m}$ ). (b, lower panels) Cone transduction by the different AAV serotypes. Co-localization of EGFP expression and PNA-lectin staining (cone sheats in red) evaluated by confocal microscopy (scale bar $=100 \mu \mathrm{m}$ ). 
injected per serotype; $n=3$ eyes untreated; at a dose of $1.0 \times 10^{10} \mathrm{GC}$ for each vector). At 6 weeks after injection, the animals were killed to perform histological analysis and to measure EGFP expression levels.

Confocal microscopy analyses of EGFP-positive areas dissected out from the most transduced retinal portion showed robust transduction of both the RPE and photoreceptor cells with both AAV2/5 and 2/8 (Figure 1b, lower panels). In addition, cells resident in the inner nuclear layer with a shape similar to Müller cells glia (cellular bodies encompassing the entire retinal thickness) were EGFP positive (Figure 2b, lower panels). To assess the ability of the AAV serotypes tested to transduce cone photoreceptors, we stained the cone outer segments with peanut agglutinin-lectin. Confocal immunofluorescence analysis revealed that cones, in addition to rods, were efficiently transduced by all AAV serotypes tested (Figure 2a, lower panels).

Fluorescence imaging of the whole-mounted retinas showed robust and homogeneous EGFP expression in the region exposed to the AAV2/5 vector, whereas fluorescence in the retinas treated with AAV 2/ 8 appeared weaker (Figure 2a, upper panels).

To quantify AAV-mediated porcine photoreceptor transduction levels, EGFP expression was measured in peeled retinas from treated $(n=4)$ and untreated eyes $(n=2)$ by western blot and normalized against a housekeeping protein ( $\beta$-tubulin; Figure $2 \mathrm{a})$. As RPE cells are carefully removed from peeled retinas, EGFP expression levels mainly reflect photoreceptor transduction following subretinal administrations of AAV vectors containing the CMV ubiquitous promoter. EGFP expression levels following AAV2/5 administration was threefold higher than AAV2/8 (Figure 1a) or 12-folds if considering values normalized with $\beta$-Gal (ratio EGFP/ $\beta$-Gal; data not shown). These data would suggest that AAV2/5 is more efficient at transducing porcine photoreceptors than the AAV2/8 serotype. To rule out the possibility that co-injection of the AAV2/1 might have interfered with AAV2/5 or AAV2/8 transduction, AAV2/5-CMV-EGFP or AAV2/8CMV-EGFP were subretinally injected either alone $(n=4$ eye per serotype; $1.0 \times 10^{10} \mathrm{GC}$ per eye); or with $1.6 \times 10^{9}$ or $1.0 \times 10^{10} \mathrm{GC}$ per eye of AAV2/1-LacZ (corresponding to $1: 1$ or 1:6, respectively, of the dose of AAV2/5 or AAV2/8; $n=2$ eye per serotype). Wholemounted stereo-microscopy fundus appearance showed similar EGFP signal between AAV2/5 and AAV2/8 when injected alone (Figure 2b), whereas AAV2/8, but not AAV2/5, showed less EGFP signal when co-injected with AAV2/1 (Figure 2a). Western blot analysis showed that AAV2/8 provided fivefold higher EGFP expression than AAV2/5 when injected without AAV2/1. In addition, AAV2/8 displayed an AAV2/1 dose-dependent decrease of retinal transduction (Figure 2b). We thus conclude that AAV2/8 is a more robust gene delivery vehicle for photoreceptors transduction than AAV2/5 (as we have previously demonstrated in mice ${ }^{15}$ ). This is in agreement with a previous study in dogs, ${ }^{17}$ which however lacked the side-by-side comparison between the two AAV serotypes that we have performed. Consistent with this observation, two additional studies have demonstrated that AAV2/8-mediated gene complementation enables significant protection from severe photoreceptor degeneration in a mouse model of $L C A^{29}$ (likely owing to the limited efficiency of photoreceptor gene transfer provided by previously tested AAV serotypes). The data presented here underscore that the photoreceptor gene transfer properties of AAV2/8 are conserved among different species.
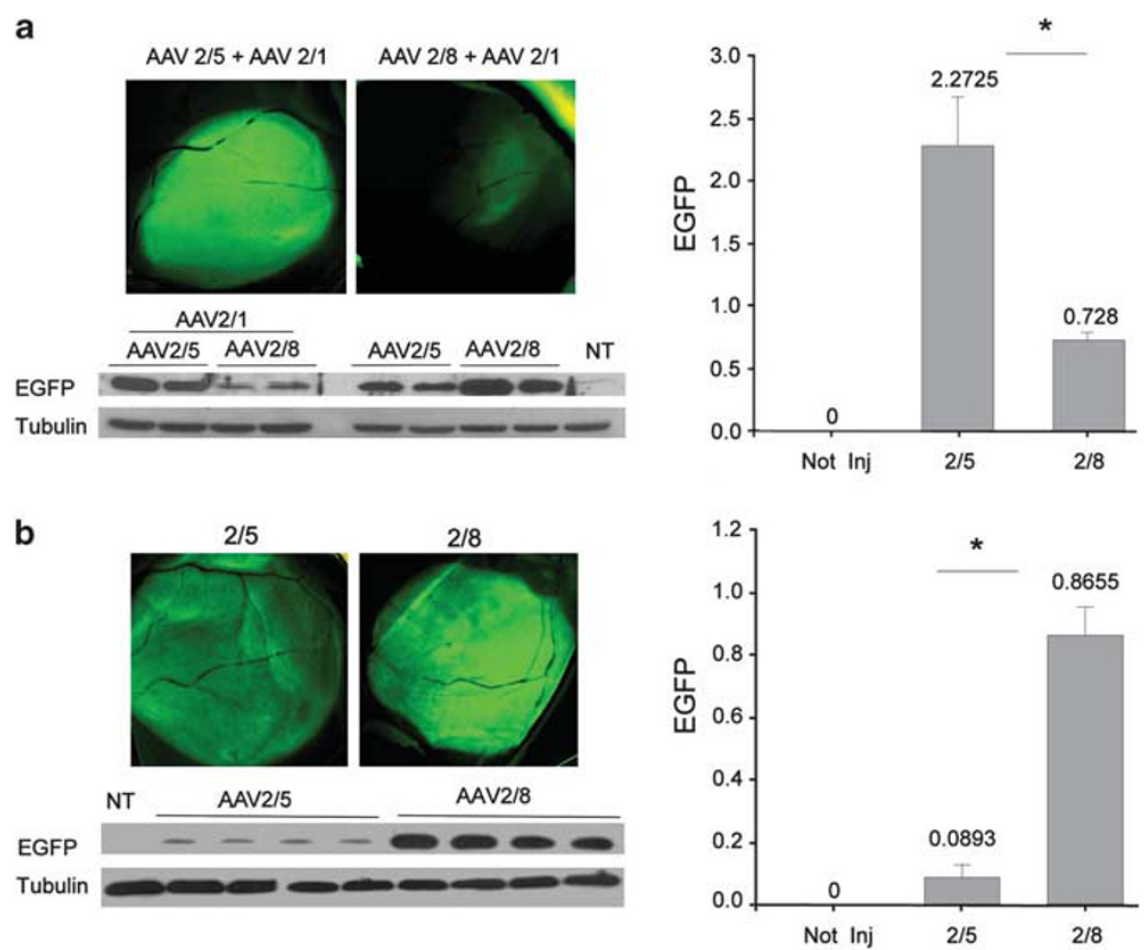

Figure 2 AAV-mediated transduction of the porcine retina. (a) Fundus fluorescence imaging of the whole-mounted retinas showed higher EGFP expression in AAV2/5- than AAV2/8 treated retinas when co-injected with AAV2/1 (Figure 1a, upper panels). (a, Lower panel) Western blot analysis with anti-EGFP (upper blot) or anti- $\beta$-tubulin (lower blot) antibodies of lysates from swine retinas transduced with AAV-2/5, $-2 / 8$ ( $n=4$ for each serotype). (a, Lower right) Histogram representing the EGFP expression levels measured by western blot. In the histogram values of EGFP (pixels inches ${ }^{-1}$ ) normalized against $\beta$-tubulin (pixels inches $^{-1}$ ) are reported ( $n=4$ eyes per group; ${ }^{*} \mathrm{AAV} 2 / 5$ vs AAV2/8: $P=0.0098$ ). (b) Fundus appearance (upper left) and quantification of the EFGP expression levels (upper right and lower panels) by western blot analysis of porcine retinas injected with AAV2/5-CMV-EGFP or AAV2/8-CMV-EGFP alone ( $n=4$ eyes per group; ${ }^{*} \mathrm{AAV} 2 / 5$ vs AAV2/8: $P=0.00014$ ). 
This result is not trivial considering other studies in which different interspecies transduction levels were observed for the same AAV serotype. $^{30-33}$

Our data also show that AAV2/1 inhibits AAV2/8 retinal transduction. This suggests that the two vectors may compete for similar receptors/co-receptors or post-entry pathways.

\section{Ubiquitous and photoreceptor-specific promoters are similarly efficient in porcine photoreceptor transduction}

To select the most efficient promoter for porcine photoreceptor transduction, we generated AAV2/5 vectors encoding EGFP under the transcriptional control of the cytomegalovirus $\left(\mathrm{CMV}^{34}\right)$, Chicken$\beta$-actin $\left(\mathrm{CBA}^{35}\right)$ ubiquitous promoters or the human rhodopsin $\left(\mathrm{RHO}^{36}\right)$ photoreceptor-specific proximal promoter (U16824 GeneBank; sequence, -800 to +6), namely AAV2/5-CMV-EGFP, AAV2/5CBA-EGFP and AAV2/5-RHO-EGFP, respectively. At 6 weeks after subretinal administration, the eyes and the retinas were collected for histological ( $n=2$ eyes per group; $n=1$ untreated) and western blot analyses of EGFP expression ( $n=3$ eyes pet group; $n=2$ untreated). As expected from previous studies in mice, the EGFP expression pattern from AAV2/5 vectors harboring the CMV or the CBA promoters ${ }^{11,13,14}$ was localized to both RPE and photoreceptors, whereas the RHO promoter restricted transgene expression to photoreceptors (Figure 3a, upper panels $\left.{ }^{13,36}\right)$. In addition, co-localization of EGFP and peanut agglutinin staining evaluated by confocal microscopy analysis demonstrated an efficient EGFP expression in cone photoreceptors independently of the promoter used (Figure 3a, lower panels). We next quantified the EGFP expression levels in the retinas treated with $\mathrm{AAV} 2 / 5$ vectors harboring different promoters by western blot analysis (Figure 3b) as described in the previous paragraph. Subretinal administration of AAV2/5 vectors containing ubiquitous (CBA or $\mathrm{CMV}$ ) and photoreceptor-specific (RHO) promoters resulted in similar EGFP expression levels with a slightly higher, although not statistically significant, EGFP/ $\beta$-Gal ratio in samples treated with the vector containing the EGFP driven by the RHO promoter (Figure 3c). Although the EGFP levels obtained using the RHO promoter faithfully represent both cone and rod photoreceptor transductions, those using the CBA and CMV promoters may additionally reflect transduction of a few contaminating RPE and inner retinal cells. The relative levels of transduction obtained in the porcine retina using the RHO promoter, compared with the ubiquitous CMV and CBA, differ from what we observed previously in the murine retinas, in which the use of the same RHO promoter element led to the highest reporter gene expression levels. The transcriptional activity of the RHO promoter is likely to be higher in rods than cones, thus resulting in higher transduction levels in the murine rod-dominant than in the porcine cone-enriched retina.

Retinal function, histopathology, neutralizing antibodies and vector biodistribution following AAV subretinal administration in pigs To assess whether AAV vector administration and/or the surgical technique applied resulted in detrimental effects on retinal function, full-field electroretinograms (ERGs) were recorded at baseline and before killing (Figure 4). Rod and cone traces were evaluated separately. Both rod and cone isolated responses of treated eyes (recorded at 6 weeks after vector delivery) showed no statistical difference compared with baseline preoperative values recorded the day of surgery (Figures $4 \mathrm{a}-\mathrm{d}$; baseline pre-treatment $n=6$, post-treatment $n=5$ ). On the contrary, in the animal with retinal detachment, the ERG was not recordable (Supplementary Figure 1). Interestingly, the

C
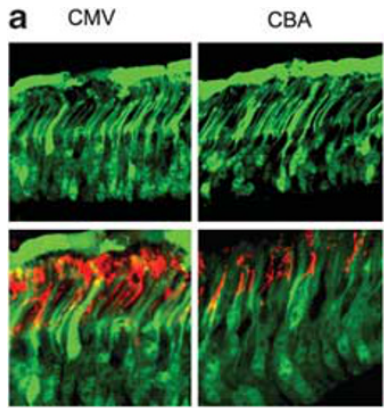

RHO

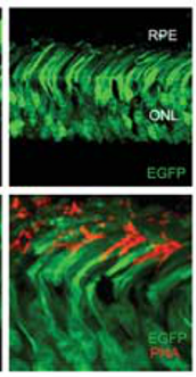

b

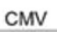

RHO

NT

Tubulin

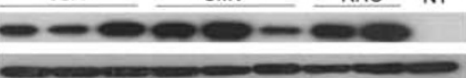

Figure 3 Subretinal injections of AAV2/5 vectors expressing EGFP from ubiquitous and photoreceptor-specific promoters. (a, Upper panels) Confocal fluorescence microscopy images ( $\times 63$ magnification) of retinal sections showing EGFP expression from cytomegalovirus (CMV), Chicken- $\beta$-actin (CBA) or human rhodopsin $(\mathrm{RHO})$ promoters (Scale bar $=100 \mu \mathrm{m})$. (a, Lower panels) Cone transduction is indicated by the co-localization of EGFP expression and PNA staining (arrows). RPE, retinal pigment epithelium; ONL, outer nuclear layer (scale bar $=100 \mu \mathrm{m}$ ). (b) Western blot analysis with anti-EGFP (upper blot) or anti- $\beta$-tubulin (lower blot) antibodies of lysates from porcine retinas transduced with AAV-2/5-EGFP driven by CMV, CBA or RHO promoters. (c) In the left histogram, values of EGFP (pixels inches ${ }^{-1}$ ) normalized against $\beta$-tubulin (pixels inches ${ }^{-1}$ ) are reported ( $n=3$ for each serotype).

Figure 4 Safety of AAV vector delivery to porcine retinas. Electroretinographic recordings at baseline (pretreatment) and at killing (post treatment). (a-d) Comparison of the tracings derived from representative eyes before and after subretinal injections of AAV vectors. The histograms show the maximum B-wave average response of all recorded eyes ( $n=6$ eyes pre-treatment; $n=5$ eyes post treatment). (a) Rod responses (dark-adapted conditions); (b) Maximal response (A-wave and B-wave) of rods and cones (dark-adapted conditions, single white flash); (c) Cone responses isolated using a bright single flash (lightadapted conditions, background white light); (d) Cone responses isolated using a flicker response (light-adapted conditions). (e) Immunofluorescence image ( $\times 20$ magnification) of retinal sections from animals injected with AAV2/5 or 2/8 and stained with glial fibrillary acidic protein (GFAP; in red) as marker of gliosis. EGFP expression (in green) shows the transduced area. RPE, retinal pigment epithelium; ONL, outer nuclear layer; INL, inner nuclear layer (scale bar $=10 \mu \mathrm{m}$ ). 
electrophysiological measurements showed high photopic and low scotopic amplitudes compared with those of NHP and humans, ${ }^{21,37}$ demonstrating a predominant contribution of cone photoreceptors to the ERG responses in porcine retinas. The ERG findings support the concept that both cone and rod photoreceptor functions were preserved after AAV2/5, and 2/8 subretinal administration.

a
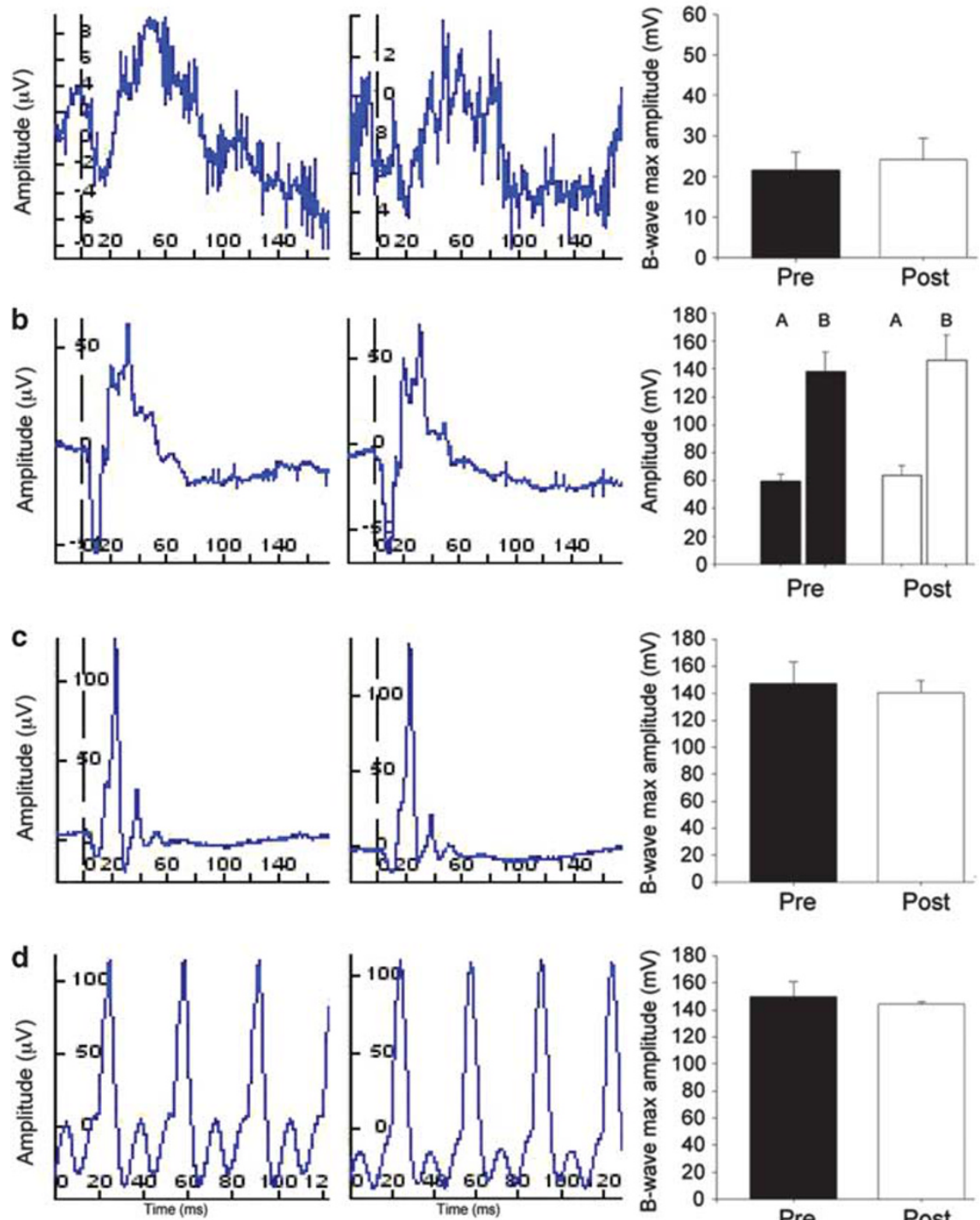

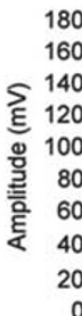

A

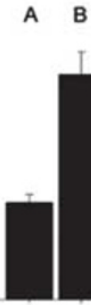

Pre

Post

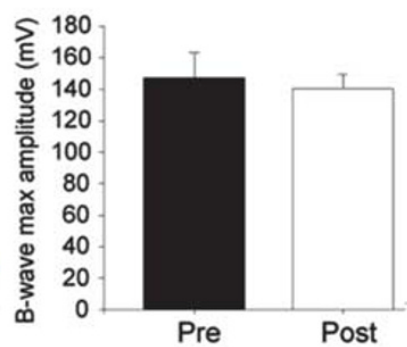

e
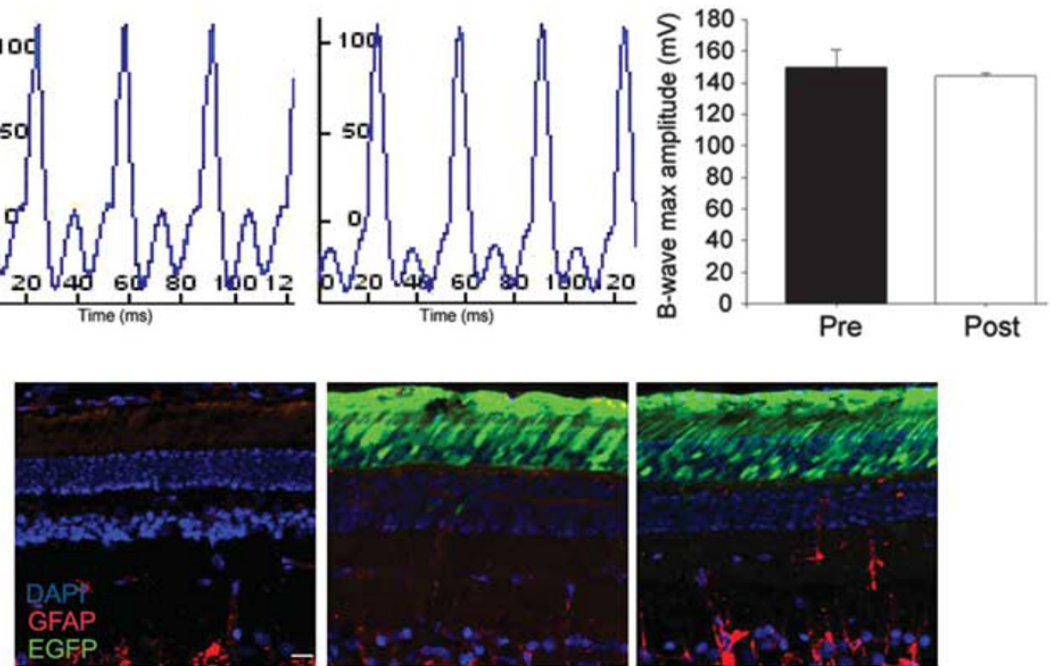

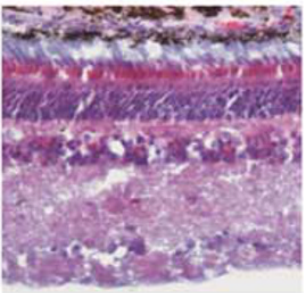

Untreated

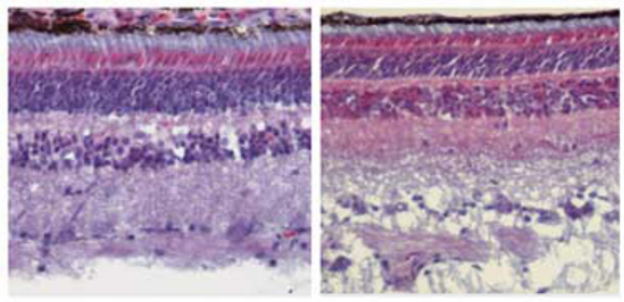

$2 / 5$

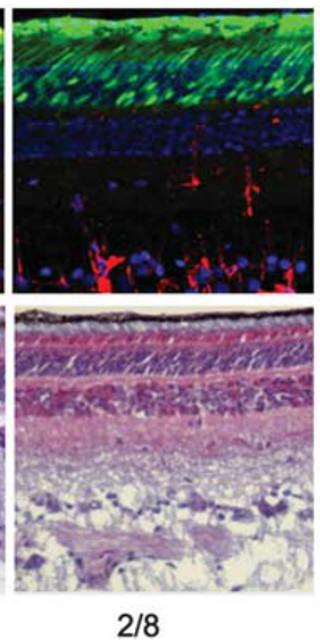

$2 / 8$ 
To investigate the presence of neutralizing antibodies (NAbs) to the AAV vector capsids, serum and anterior chamber fluid samples were collected at the day of surgery and at killing (at 6 weeks after injection) from all animals injected subretinally with AAV vectors. ${ }^{19,38}$ Out of 21 animals tested, including those with retinal detachment (AAV2/5 $n=11$ and AAV2/8 $n=5$ ) only 3 animals developed NAbs in the serum (one per AAV serotype injected) after treatment, whereas a modest increase of NAb levels was detected in the anterior chamber fluids of one animal (P19 left eye) with retinal detachment (Supplementary Figure 3).

Histopathological analyses (hematoxilin and eosin staining) of retinal sections evaluated at killing, revealed normal retinal histology, including lack of inflammatory cell infiltrates in the eyes treated with the different AAV vectors tested (Figure 4e, lower panels). Histopathological analysis performed on one eye with retinal detachment at ophthalmological visit showed RPE atrophy, hypertrophy of melanocytes and RPE cells, and extensive degeneration of the outer nuclear layer and photoreceptor outer segments (Supplementary Figure 1).

To assess whether AAV injections induced gliosis in the transduced retinas, we performed glial fibrillary acidic protein (red) staining of retinal sections at killing. As shown in Figure 4e, no significant differences were observed among the retinal sections derived from treated and/or untreated retinas. To evaluate whether either the surgical procedure or the AAV-mediated transduction induce photoreceptor apoptosis in the cells, TUNEL staining was performed on retinal sections of treated and untreated eyes. This analysis was also performed from retinal sections of one eye with retinal detachment (Supplementary Figure 2). We did not observe apoptotic nuclei (TUNEL stained; red), suggesting that the degeneration caused by retinal detachment occurred before collecting.

To determine AAV biodistribution following subretinal delivery to the porcine retina, we extracted HIRT DNA from the following: the optic nerve, optic chiasm, visual thalamus, visual occipital cortex, muscle (tongue), lung, liver, spleen, ovaries and peripheral blood mononuclear cells. Samples were analyzed by real-time PCR $(n=11$ animals) with primers specific for the AAV genome. Table 1 reports $\mathrm{AAV} 2 / 5$ or AAV2/8 biodistribution in the retina and in different areas of the brain, including one animal with retinal detachment (P3; Table 1, asterisk). The results support the presence of AAV genomes in all retinas and in two optic nerves and not in the brain (Table 1) or the other extraocular tissues analyzed (data not shown). These results indicate that the risk of the vector spreading outside the treated tissue is minimal at the AAV doses used in this study. Previous studies reported the presence of $\mathrm{AAV}$ vector genomes in the lateral geniculate nucleus of NHP that received AAV2/2 subretinal injections, ${ }^{20}$ whereas vector DNA, EGFP mRNA and EGFP protein were found in neurons of the lateral geniculate nucleus contralateral to the injected eye and in several parts of the brain after subretinal delivery of AAV2/8 in dogs, ${ }^{17}$ suggesting that trans-synaptic transport of vector particles had occurred. In the present study, the presence of AAV genomes in two optic nerves associated with the lack of genomes in the thalamus and occipital cortex favors the hypothesis that accidental leakage of vector solution in the vitreous during subretinal infusion may result in infections of retinal ganglion cells. Altogether, these data support the safety of AAV vectors subretinal administration to the porcine eye.

The side-by-side comparison of the porcine retinal transduction characteristics, following subretinal administration of AAV2/5 and AAV2/8 vectors serotypes herein reported shows that: (1) both vectors efficiently transduce photoreceptors and RPE; (2) AAV2/8 transduces at higher levels than AAV2/5 the porcine cone-enriched outer retina; and (3) both the ubiquitous and photoreceptor-specific promoters tested here allow similar levels of porcine retinal transduction. Given the high cone/rod ratio of the porcine retina, and the genetic and physiological similarities between pigs and humans, our data may affect the development of gene-based therapies for rare and common retinal diseases affecting both rod and cone photoreceptors. In addition, the pig may be considered as a valuable bridging animal model to assess tropism and safety of retinal gene transfer before proceeding to the formal good laboratory practice toxicity studies in NHP, typically required by regulatory agencies for a human clinical trial.

\section{MATERIALS AND METHODS}

\section{Generation of the plasmid constructs}

For the production of AAV-encoding EGFP with different promoters, pAAV2. 1-CMV-EGFP, ${ }^{39}$ pAAV2.1-CBA-EGFP and pAAV2.1-RHO-EGFP plasmids were used. pAAV2.1-RHO-EGFP and pAAV2.1-CBA-EGFP were obtained by exchanging the CMV promoter of PAAV2.1-CMV-EGFP with the RHO (-800 to +6 relative to the transcriptional start site; GenBank accession number U16824) or the CBA promoters. The RHO promoter was PCR amplified from the human genomic DNA to insert the NheI and NotI sites at the $5^{\prime}$ and $3^{\prime}$ ends, respectively, using the following primers: NheI-RHO-Forward, $5^{\prime}$-AATTATGCT AGCAGATCTTCCCCACCTAGC-3' and NotI-RHO-Reverse, 5'-ATTAATGC GGCCGCGGATGACTCTGGGTTCTG-3'. The PCR product was then digested with NheI and NotI and cloned in AAV2.1-CMV-EGFP after removing the CMV promoter. The CBA promoter was amplied from the pCAGGS plasmid (a kind gift by M Studer, TIGEM, Naples, Italy) by PCR to insert the NcoI and PstI sites at the $5^{\prime}$ and $3^{\prime}$ ends, respectively. The PCR product was then cloned

Table 1 Biodistribution following subretinal delivery in pigs (GC $\mathrm{mdg}^{-1}$ )

\begin{tabular}{|c|c|c|c|c|c|c|c|}
\hline & & Treatment & Retina & Optic nerve & Chiasm & Thalamus & Visual cortex \\
\hline R1 & Left & AAV2/8 & $6.771 \pm 0.205$ & ND & ND & ND & ND \\
\hline R2 & Left & AAV2/8 & Not measured & ND & ND & ND & ND \\
\hline R3 & Left & AAV2/8 & $2.340 \pm 0.043$ & ND & ND & ND & ND \\
\hline R4 & Left & AAV2/8 & $2.552 \pm 0.126$ & ND & ND & ND & ND \\
\hline \multirow[t]{2}{*}{ R5 } & Left & AAV2/5 & $4.952 \pm 0.379$ & ND & ND & ND & ND \\
\hline & Right & AAV2/5 & $34.344 \pm 0.094$ & $0.491 \pm 0.005$ & ND & ND & ND \\
\hline \multirow[t]{2}{*}{ R6 } & Left & AAV2/5 & $58.104 \pm 1.480$ & $0.444 \pm 0.002$ & ND & ND & ND \\
\hline & Right & AAV2/5 & Not measured & ND & ND & ND & ND \\
\hline \multirow[t]{3}{*}{ P3 } & Left & AAV2/5-RHO & Not measured & ND & ND & ND & ND \\
\hline & Right* & AAV2/5-CMV & Not measured & ND & ND & ND & ND \\
\hline & Right & Not Injected & ND & ND & ND & ND & ND \\
\hline
\end{tabular}

Abbreviations: GC, genome copies; mdg, molecule of diploid genome; nd, not detected. Asterisk indicates eye with a retinal detachment. 
into pAAV2.1-CMV-EGFP (digested with NcoI and PstI) downstream of the CMV enhancer. The pAAV2.1-CMV-LacZ plasmid has been previously described. ${ }^{39}$

\section{AAV vector production}

AAV vectors were produced by the TIGEM AAV Vector Core (Naples, Italy) using the pAAV2.1-CMV-EGFP, -CBA-EGFP, -RHO-EGFP and -CMV-LacZ plasmids. Recombinant AAV2/5, and 2/8 viruses were produced by triple transfection of 293 cells followed by cesium chloride purication. ${ }^{11}$ For each viral preparation, physical titers (in (GC) per ml) were determined by both PCR quantication using TaqMan (PerkinElmer Life and Analytical Sciences, Boston, MA, USA) and dot-blot analysis. ${ }^{40}$

\section{Animals and subretinal administration of AAV vectors}

All the experiments regarding animals were conducted according to the rules approved by the Italian Institution for animal research. The Large White pigs used in our study were registered as purebred in the LW Herd Book of the Italian National Pig Breeders' Association. Pigs were starved overnight leaving water ad libitum.

Before surgery, eyes were dilated with topical 2.5\% phenylephrine (Bausch \& Lomb Ltd., London, UK). The surgical procedure envisaged two-port sclerotomy, one for the light source and the other for the injection. Briefly, one drop of $5 \%$ povidone iodine solution (Betadine, Meda Pharma, Milano, Italy) was applied to the ocular surface and in the cul-de-sac for $3 \mathrm{~min}$, followed by insertion of lid speculum. The procedure started with a transconjunctival scleral tunnel incision via pars plana parallel to the corneoscleral limbus at $3.5 \mathrm{~mm}$. The angle insertion of 20- or 23-gauge stiletto blade (Alcon, Fort Worth, TX, USA) was performed for every case to facilitate the efficiency of self-sealing. Subsequently the light fiber attached to the vitrectomy unit (ACCURS vitrectomy machine, Alcon) and either a 38-gauge (Alcon) or extendible 41-gauge subretinal injection needles (DORC, Zuidland, the Netherlands) were, respectively, inserted through the two conjunctival incisions and into the two scleral tunnels. Therefore, illuminating the posterior pole with the light fiber and without vitreous removal, the injection in the subretinal space in the nasal area was performed with a 1-ml syringe connected to the subretinal needles, slowly and under direct observation with a stereoscopic microscope. At the end of surgery, paracentesis with removal of $0.1 \mathrm{ml}$ of aqueous humor was performed using a $1-\mathrm{ml}$ syringe with 30 -gauge needle to collect anterior chamber fluid sample (preoperative) and to avoid an increase in intraocular pressure. Animals underwent handy slit-lamp biomicroscopy and indirect ophthalmoscopy before and immediately after injection, within 3 days after surgery and at killing at 6 weeks post-vector administration.

\section{Anesthetic procedure}

Premedication included intramuscular injection of azaperone $\left(2 \mathrm{mg} \mathrm{kg}^{-1}\right.$ of Stresnil, Janssen-Cilag SpA, Milano, Italy). Pigs were left undisturbed for 20 mins and then received a second intramuscular injection of ketamine (20 $\mathrm{mg} \mathrm{kg}^{-1}$ of Ketavet 100, Intervet Productions S.r.l., Segrate, Italy), acepromazine $\left(100 \mu \mathrm{g} \mathrm{kg}^{-1}\right.$ of Prequillan, ATI S.r.l., Bologna, Italy) and atropine $\left(0.04 \mathrm{mg} \mathrm{kg}^{-1}\right.$ of Atropina solfato, ATI S.r.l.). An auricular vein was catheterized by the insertion of an over-the needle catheter (Delta Ven1, DELTA MED S.r.l., Mantova, Italy) and a saline solution was infused i.v. at a rate of $10 \mathrm{ml} \mathrm{kg}^{-1}$ per hour. General anesthesia was induced and maintained by intermittent i.v. boluses of ketamine ( $5 \mathrm{mg} \mathrm{kg}^{-1}$ of Ketavet 100, Intervet Productions S.r.l.) and diazepam $\left(0.5 \mathrm{mg} \mathrm{kg}^{-1}\right.$ of Diazepam $0.5 \%$, Intervet Productions S.r.l.) during the whole ERG procedure. Animals were then moved to the surgery room where they were orotracheally intubated, following an i.v. bolus of sodium thiopental (5-10 $\mathrm{mg} \mathrm{kg}^{-1}$ of Pentothal Sodium, Intervet Productions S.r.l.). During the surgical procedure, anesthesia was maintained with isoflurane in $100 \%$ oxygen and pigs were mechanically ventilated (Falco 201, Siare Hospital, Bologna, Italy) setting a tidal volume and a respiratory rate to maintain end-tidal $\mathrm{PCO}_{2}$ between 35 and $45 \mathrm{~mm} \mathrm{Hg}$

\section{Fundus photography}

Eyecups were collected, fixed by immersion in $4 \%$ paraformaldehyde for $12 \mathrm{~h}$ and then rinsed briefly with phosphate-buffered saline (Sigma, St Louis, MO, USA). Cornea and lenses were removed and fundus photographs were taken with an Axioplan microscope (Carl Zeiss, Oberkochen, Germany) in bright field and under fluorescence light to evaluate the EGFP expressing area.

\section{Sampling of the anterior chamber fluid, vitreous and retina}

Pigs were anesthetized as described above and killed, following ERG procedure and blood sampling, with an i.v. administration of $10 \mathrm{ml}$ of Tanax (Intervet Italia, Segrate, Italy), and their eyeballs were collected using a pair of scissors to cut the muscles and the optic nerve. Each eye was placed in a petridish filled with phosphate-buffered saline and rinsed twice. The conjunctiva and tendons were cleaned off the globe and a 33-gauge needle connected to a syringe was used to aspirate the anterior chamber liquid. The fluid was placed in an Eppendorf tube (Eppendorf, Hamburg, Germany) for the detection of the NAbs and immediately frozen in liquid nitrogen. A scalpel was used to dig into the eye and vannas scissors (WPI, Sarasota, FL, USA) were used to separate the anterior chamber including the iris and lens from the eyecup. Special care was given to avoid compressing the eye. To prevent any suction of the retina, the vitreous was carefully aspirated by using a syringe without a needle, placed in an Eppendorf tube (Eppendorf) and immediately frozen in liquid nitrogen. The eyecup was then cut in 4-5 parts and the retina was detached from the RPE using a scraper. The attachment to the optic nerve was then severed and the retina was collected with blunt forceps, placed in an Eppendorf tube (Eppendorf) and immediately frozen.

\section{Histological analysis}

Pigs were killed and their eyeballs were collected and fixed overnight by immersion in $4 \%$ paraformaldehyde. The eyeballs were cut so that the lens and vitreous could be removed, leaving the eyecups in place. Tissue-freezing medium (OCT matrix, Kaltek, Padua, Italy) embedding was performed by infiltrating swine eyecups with $30 \%$ sucrose. Paraffin preparation of samples was performed by dehydratation before the standard embedding procedure. For each eye, $200-300$ serial sections ( $14 \mu \mathrm{m}$ thick) were cut along the horizontal meridian, and the sections were progressively distributed on glass slides so that each slide contained 4-6 sections representative of different levels of the eye. The sections were stained with 4',6'-diamidino-2-phenylindole (VectashieldVector Laboratories Inc., Peterborough, UK) or with hematoxylin and eosin (Richard-Allen Scientific, Kalamazoo, MI, USA; Aldrich, Milan, Italy) according to the standard protocol, and retinal histology images were obtained with an Axioplan microscope (Carl Zeiss) with $\times 20$ magnification.

\section{PNA, GFAP and TUNEL immunolabeling on retinal sections}

Sections were rinsed in phosphate-buffered saline and incubated for $1 \mathrm{~h}$ in $10 \%$ fetal bovine serum in PBT $(0.1 \%$ Triton $100,0.1 \%$ bovine serum albumin in phosphate-buffered saline). The incubation with biotinylated peanut agglutinin (PNA, $400 \mathrm{mg} \mathrm{ml}^{-1}$ in PBT; Vector Laboratories Inc.) or with glial fibrillary acidic protein, 1:500 in PBT (DakoCytomation, Glostrup, Denmark) was performed overnight at $4{ }^{\circ} \mathrm{C}$. The sections were then washed three times with phosphate-buffered saline and incubated with Alexa Fluor 568-coupled streptavidin (1:250 in PBT, Molecular Probes, Invitrogen, Carlsbad, CA, USA) for $1 \mathrm{~h}$ at room temperature. After a final wash, sections were mounted with $4^{\prime}, 6^{\prime}$ diamidino-2-phenylindole (Vectashield), and images were obtained with a confocal microscope Leica DMIRE2 and Leica confocal software (Leica Microsystem, Wetzlar, Germany) with $\times 63$ magnification. Apoptotic nuclei were detected by TdT-dUTP terminal nick-end labeling kit (TUNEL, Roche Diagnostics, Monza, Italy) according to the manufacturer's protocol and images were obtained with an Axioplan microscope (Carl Zeiss) with $\times 20$ magnification.

\section{EGFP and $\beta$-Gal quantification}

Collection of retinas was performed as described above (see Histological analysis section). EGFP expression levels were measured in retinal lysates by western blotting in $100 \mu \mathrm{g}$ of total retinal lysate. Band intensity was estimated using the Image software (NIH, version 1.41) and values (pixels inches ${ }^{-1}$ ) corresponding to EGFP were normalized on endogenous $\beta$-tubulin levels.

$\beta$-Gal expression levels were measured by enzyme-linked immunosorbant assay (Roche Diagnostics) according to the manufacturer's instructions and normalized per microgram of retinal total lysates used. Histograms reported the normalized EGFP values or the EGFP/ $\beta$-Gal ratio. 


\section{DNA preparation and biodistribution study}

The spreading of vector DNA in tissues was determined in samples collected at killing. Tissues (retinas, optic nerve, optic chiasm, visual thalamus, visual cortex, muscle (tongue), lung, liver, spleen, gonads, peripheral blood mononuclear cells) were collected with appropriate precautions to avoid any crosscontamination and immediately frozen in liquid nitrogen. DNA extraction was performed using HIRT solution, as previously reported, ${ }^{41}$ and immediately transferred to storage in which it remained until the time of analysis. Total copies of the AAV genome in tissue samples (vector genome copy number per diploid genome GC (molecule per diploid genome) $\mathrm{mdg}^{-1}$ ) were quantified by real-time PCR using an ABI 7700 Sequence Detection System (Applied Biosystems, Foster City, CA, USA) and analyzed using the SDS 2.0 software (Software Diversified Services, Spring Lake Park, MN, USA). Real-time PCR analysis was performed on $100 \mathrm{ng}(\sim 16000$ diploid genomes considering an average weight for a single mdg of $6.6 \mathrm{pg}$ ) of HIRT-extracted genomic DNA ${ }^{42}$ using the following set of TaqMan primers/probe (PE Biosystems, Foster City, CA, USA): forward 5'-TCTAGTTCGCAGCCATCTGTTGT-3'; reverse 5'-TGG GAGTGGCACCTTCCA-3'; probe: 5'-CCCCCGTGCCTTCCTTGACC-3', specific for the bovine growth hormone polyA. A calibration curve was prepared using a linear AAV2-CMV-EGFP genome spanning from $5 \times 10^{1}$ $\left(\sim 0.01: 1 \mathrm{GC} \mathrm{mdg}^{-1}\right)$ to $5 \times 10^{7}\left(\sim 3000: 1 \mathrm{GC} \mathrm{mdg}^{-1}\right)$ as template. Real-time PCR reaction was carried out in $20 \mu \mathrm{l}$ final volume using the following program: $1 \mathrm{~min}$ at $94^{\circ} \mathrm{C}, 1 \mathrm{~min}$ at $60^{\circ} \mathrm{C}, 1 \mathrm{~min}$ at $72^{\circ} \mathrm{C}$ for 40 cycles; the amplification cycles were anticipated by a denaturing step $\left(5 \mathrm{~min}\right.$ at $\left.95^{\circ} \mathrm{C}\right)$.

Identification of neutralizing antibodies against the virus capsids The presence of NAbs against the virus capsid used in this study (AAV2/5 and AAV2/8) was assessed on samples collected at the day of injection (preoperative) and the day of killing (postoperative), as previously described. ${ }^{19}$ The samples analyzed included the serum and the anterior chamber fluid. The NAb titer was defined as the highest dilution that allowed EGFP to be produced to levels corresponding to $50 \%$ of those found in control samples (in which serum had not been added to the virus before infection).

\section{Electroretinogram recordings}

Bilateral ERG evaluations were performed using a portable ERG unit (HMsERG model 1000, RetVet, Columbia, MO, USA), with the mini-ganzfeld dome positioned approximately $1 \mathrm{~cm}$ from the right tested eye. Each ERG session consisted of scotopic and photopic ERGs in accordance with the International Society for Clinical Electrophysiology of Vision, recommended by the European College of Veterinary Ophthalmology, primarily for evaluation of rod and cone function. ${ }^{37}$ This protocol is pre-programmed on the ERG unit and is executed automatically upon initiation of the ERG session by the examiner. Platinum subdermal needle electrodes (model E2, Grass Instrument Division, Astro-Med, West Warwick, RI, USA) were used for the ground electrode, positioned on the occipital crest and for the reference electrode, positioned $3 \mathrm{~cm}$ from the lateral canthus, close to the base of the right ear. An active contact lens electrode (ERG-Jet, Universo Plastique, LKC Technologies, Gaithersburg, MD, USA) was placed on the cornea after instillation of one drop of 2\% methylcellulose (Methocel, Ciba Vision, Munich, Germany). Rod responses were measured in dark-adapted conditions with a light intensity of $10 \mathrm{mcd} . \mathrm{s} \mathrm{m}^{-2}$, with 10 flashes of $2 \mathrm{~s}$ each and a $20 \mathrm{~ms}$ flash lasting $180 \mathrm{~ms}$. The maximal response of rods and cones was measured in dark-adapted conditions with a light intensity of $10 \mathrm{~cd} . \mathrm{s} \mathrm{m}^{-2}$, with four flashes of $20 \mathrm{~s}$ each and a $20 \mathrm{~ms}$ flash lasting $180 \mathrm{~ms}$. Cone responses were isolated in light-adapted conditions with a background white light of $30 \mathrm{~cd} . s$, a light intensity of $3 \mathrm{~cd} . \mathrm{s} \mathrm{m}^{-2}$, with 32 flashes of $0.5 \mathrm{~s}$ each and a $20 \mathrm{~ms}$ flash lasting $180 \mathrm{~ms}$. Cone responses using a flicker ERG were measured in light-adapted conditions with a light intensity of $10 \mathrm{~cd} . \mathrm{s} \mathrm{m}^{-2}$, with 128 flashes at $30 \mathrm{~Hz}$.

\section{CONFLICT OF INTEREST}

The authors declare no conflict of interest.

\section{ACKNOWLEDGEMENTS}

We are grateful to Luciana Borrelli (TIGEM) for critical revision of the manuscript. We thank the TIGEM AAV vector Core for providing the AAV vector preps used in this study. This work was supported by the Telethon Foundation (grant TIGEM P21), the Milton \& Steinbach Fund, the National Institute of Health (grant 1R01EY015136-01), the Italian Ministry of Agriculture (grant D.M.589/7303/04) and by the European Union within Seventh (AAVEYE and TREATRUSH contract number 223445 and 242013, respectively) Framework Program.

1 Cremers FP, van den Hurk JA, den Hollander Al. Molecular genetics of Leber congenital amaurosis. Hum Mol Genet 2002; 11: 1169-1176.

2 Bainbridge JW, Smith AJ, Barker SS, Robbie S, Henderson R, Balaggan K et al. Effect of gene therapy on visual function in Leber's congenital amaurosis. N Engl J Med 2008; 358: 2231-2239.

3 Cideciyan AV, Aleman TS, Boye SL, Schwartz SB, Kaushal S, Roman AJ et al. Human gene therapy for RPE65 isomerase deficiency activates the retinoid cycle of vision but with slow rod kinetics. Proc Natl Acad Sci USA 2008; 105: 15112-15117.

4 Cideciyan AV, Hauswirth WW, Aleman TS, Kaushal S, Schwartz SB, Boye SL et al. Human RPE65 gene therapy for Leber congenital amaurosis: persistence of early visual improvements and safety at 1 year. Hum Gene Ther 2009; 20: 999-1004.

5 Cideciyan AV, Hauswirth WW, Aleman TS, Kaushal S, Schwartz SB, Boye SL et al. Vision 1 year after gene therapy for Leber's congenital amaurosis. N Engl J Med 2009; 361: 725-727.

6 Hauswirth WW, Aleman TS, Kaushal S, Cideciyan AV, Schwartz SB, Wang $L$ et al. Treatment of leber congenital amaurosis due to RPE65 mutations by ocular subretinal injection of adeno-associated virus gene vector: short-term results of a phase I trial. Hum Gene Ther 2008; 19: 979-990.

7 Maguire AM, Simonelli F, Pierce EA, Pugh Jr EN, Mingozzi F, Bennicelli J et al. Safety and efficacy of gene transfer for Leber's congenital amaurosis. N Engl J Med 2008; 358: 2240-2248.

8 Dejneka NS, Surace EM, Aleman TS, Cideciyan AV, Lyubarsky A, Savchenko A et al. In utero gene therapy rescues vision in a murine model of congenital blindness. Mol Ther 2004; 9: 182-188.

9 Acland GM, Aguirre GD, Ray J, Zhang Q, Aleman TS, Cideciyan AV et al. Gene therapy restores vision in a canine model of childhood blindness. Nat Genet 2001; 28: 92-95.

10 Wolfensberger MFMTJ. The Retinal Pigment Epithelium: Function and Disease, 1st edn. Oxford University Press: USA, 1998.

11 Auricchio A, Kobinger G, Anand V, Hildinger M, O'Connor E, Maguire AM et al. Exchange of surface proteins impacts on viral vector cellular specificity and transduction characteristics: the retina as a model. Hum Mol Genet 2001; 10: 3075-3081.

12 Stieger K, Lheriteau E, Moullier P, Rolling F. AAV-mediated gene therapy for retinal disorders in large animal models. Ilar J 2009; 50: 206-224.

13 Allocca M, Mussolino C, Garcia-Hoyos M, Sanges D, lodice C, Petrillo M et al. Novel adeno-associated virus serotypes efficiently transduce murine photoreceptors. J Virol 2007; 81: 11372-11380.

14 Lebherz C, Maguire A, Tang W, Bennett J, Wilson JM. Novel AAV serotypes for improved ocular gene transfer. J Gene Med 2008; 10: 375-382.

15 Natkunarajah M, Trittibach P, McIntosh J, Duran Y, Barker SE, Smith AJ et al. Assessment of ocular transduction using single-stranded and self-complementary recombinant adeno-associated virus serotype 2/8. Gene Ther 2008; 15: 463-467.

16 Alexander JJ, Umino Y, Everhart D, Chang B, Min SH, Li Q et al. Restoration of cone vision in a mouse model of achromatopsia. Nat Med 2007; 13: 685-687.

17 Stieger K, Colle MA, Dubreil L, Mendes-Madeira A, Weber M, Le Meur G et al. Subretinal delivery of recombinant AAV serotype 8 vector in dogs results in gene transfer to neurons in the brain. Mol Ther 2008; 16: 916-923.

18 Weber M, Rabinowitz J, Provost N, Conrath H, Folliot S, Briot D et al. Recombinant adeno-associated virus serotype 4 mediates unique and exclusive long-term transduction of retinal pigmented epithelium in rat, dog, and nonhuman primate after subretinal delivery. Mol Ther 2003; 7: 774-781.

19 Bennett J, Maguire AM, Cideciyan AV, Schnell M, Glover E, Anand V et al. Stable transgene expression in rod photoreceptors after recombinant adeno-associated virus-mediated gene transfer to monkey retina. Proc Natl Acad Sci USA 1999; 96: 9920-9925

20 Jacobson SG, Boye SL, Aleman TS, Conlon TJ, Zeiss CJ, Roman AJ et al. Safety in nonhuman primates of ocular AAV2-RPE65, a candidate treatment for blindness in Leber congenital amaurosis. Hum Gene Ther 2006; 17: 845-858.

21 Lotery AJ, Yang GS, Mullins RF, Russell SR, Schmidt M, Stone EM et al. Adenoassociated virus type 5 : transduction efficiency and cell-type specificity in the primate retina. Hum Gene Ther 2003; 14: 1663-1671.

22 Mancuso K, Hauswirth WW, Li Q, Connor TB, Kuchenbecker JA, Mauck MC et al. Gene therapy for red-green colour blindness in adult primates. Nature 2009; 461 (7265): 784-787.

23 Curcio $\mathrm{CAH}$, Hendrickson AE. Organization and development of the primate photoreceptor mosaic. In: Prog Retin Res, vol. 10, 1991, pp 89-120.

24 Hendrickson A, Hicks D. Distribution and density of medium- and shortwavelength selective cones in the domestic pig retina. Exp Eye Res 2002; 74: 435-444.

25 Prince JH, Diesem CD, Eglitis I, Ruskell GL. The pig. In: Thomas CC, Springfield I (ed) Anatomy and Histology of the Eye and Orbit in Domestic Animals 1960, pp 210-230. 
26 Li ZY, Wong F, Chang JH, Possin DE, Hao Y, Petters RM et al. Rhodopsin transgenic pigs as a model for human retinitis pigmentosa. Invest Ophthalmol Vis Sci 1998; 39: 808-819.

27 Petters RM, Alexander CA, Wells KD, Collins EB, Sommer JR, Blanton MR et al. Genetically engineered large animal model for studying cone photoreceptor survival and degeneration in retinitis pigmentosa. Nat Biotechnol 1997; 15: 965-970.

28 Ruiz-Ederra J, Garcia M, Hernandez M, Urcola H, Hernandez-Barbachano E, Araiz J et al. The pig eye as a novel model of glaucoma. Exp Eye Res 2005; 81: 561-569.

29 Sun X, Pawlyk B, Xu X, Liu X, Bulgakov OV, Adamian M et al. Gene therapy with a promoter targeting both rods and cones rescues retinal degeneration caused by AIPL1 mutations. Gene Therapy 2010; 17: 117-131.

30 Davidoff AM, Gray JT, Ng CY, Zhang Y, Zhou J, Spence Y et al. Comparison of the ability of adeno-associated viral vectors pseudotyped with serotype 2,5 , and 8 capsid proteins to mediate efficient transduction of the liver in murine and nonhuman primate models. Mol Ther 2005; 11: 875-888.

31 Gao GP, Alvira MR, Wang L, Calcedo R, Johnston J, Wilson JM. Novel adeno-associated viruses from rhesus monkeys as vectors for human gene therapy. Proc Natl Acad Sci USA 2002; 99: 11854-11859.

32 Gao GP, Lu Y, Sun X, Johnston J, Calcedo R, Grant R et al. High-level transgene expression in nonhuman primate liver with novel adeno-associated virus serotypes containing self-complementary genomes. J Virol 2006; 80: 6192-6194.

33 Ding W, Zhang L, Yan Z, Engelhardt JF. Intracellular trafficking of adeno-associated viral vectors. Gene Therapy 2005; 12: 873-880.

34 Grant CA, Ponnazhagan S, Wang XS, Srivastava A, Li T. Evaluation of recombinant adeno-associated virus as a gene transfer vector for the retina. Curr Eye Res 1997; 16: 949-956.

35 Sawicki JA, Morris RJ, Monks B, Sakai K, Miyazaki J. A composite CMV-IE enhancer/ beta-actin promoter is ubiquitously expressed in mouse cutaneous epithelium. Exp Cell Res 1998; 244: 367-369.
36 Flannery JG, Zolotukhin S, Vaquero MI, LaVail MM, Muzyczka N, Hauswirth WW. Efficient photoreceptor-targeted gene expression in vivo by recombinant adenoassociated virus. Proc Natl Acad Sci USA 1997; 94: 6916-6921.

37 Marmor MF, Holder GE, Porciatti V, Trick GL, Zrenner E. Guidelines for basic pattern electroretinography. Recommendations by the International Society for Clinical Electrophysiology of Vision. Doc Ophthalmol 1995; 91: 291-298.

38 Lebherz C, Auricchio A, Maguire AM, Rivera VM, Tang W, Grant RL et al. Long-term inducible gene expression in the eye via adeno-associated virus gene transfer in nonhuman primates. Hum Gene Ther 2005; 16: 178-186.

39 Auricchio A, Hildinger M, O'Connor E, Gao GP, Wilson JM. Isolation of highly infectious and pure adeno-associated virus type 2 vectors with a single-step gravity-flow column. Hum Gene Ther 2001; 12: 71-76.

40 Drittanti L, Rivet C, Manceau P, Danos O, Vega M. High throughput production, screening and analysis of adeno-associated viral vectors. Gene Ther 2000; 7: 924-929.

41 Tessitore A, Faella A, O’Malley T, Cotugno G, Doria M, Kunieda T et al. Biochemical, pathological, and skeletal improvement of mucopolysaccharidosis VI after gene transfer to liver but not to muscle. Mol Ther 2008; 16: 30-37.

42 Gao G, Qu G, Burnham MS, Huang J, Chirmule N, Joshi B et al. Purification of recombinant adeno-associated virus vectors by column chromatography and its performance in vivo. Hum Gene Ther 2000; 11: 2079-2091.

This work is licensed under the Creative Commons Attribution-NonCommercial-No Derivative Works 3.0

Unported License. To view a copy of this license, visit http:// creativecommons.org/licenses/by-nc-nd/3.0/

Supplementary Information accompanies the paper on Gene Therapy website (http://www.nature.com/gt) 